FOURIER SERIES AND THE FOURIER INTEGRAL

by

BILLY GENE CLAYBROOK

B. S., Missouri State Teachers College, 1964

\title{
A MASTER'S REPORT
}

submitted in partial fulfillment of the

requirements for the degree

MASTER OF SCIENCE

Department of Mathematics

KANSAS STATE UNIVERSITY

Manhattan, Kansas

1966

Approved by:

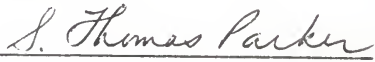


LD

2668

R4

1966

6622

C. 2

TABLE OF CONTENTS

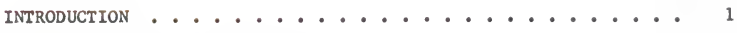

FOURIER SERIES OF A FUNCTION ................... 3

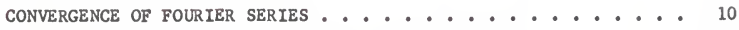

DIFFERENTIATION AND INTEGRATION OF FOURIER SER IES . . . . 15

THE FOURIER INTEGRAL .................... 20

APPLICATION OF FOURIER SERIES AND INTEGRALS ......... 30

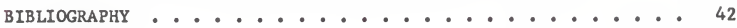

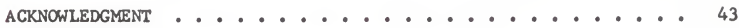




\section{INTRODUCTION}

Attention was drawn to trigonometric series in the middle of the eighteenth century when various mathematicians were studying the vibrations of stretched strings. The mathematical theory of such vibrations comes down to the problem of solving the partial differential equation $\frac{\partial^{2} u}{\partial t^{2}}=b^{2} \frac{\partial^{2} u}{\partial x^{2}}$. The general form of a trigonometric series, involving both sines and cosines, arises naturally in connection with another physical problem, that of conduction of heat. It was in the course of his study of this problem that J. B. Fourier announced in 1807 that an arbitrary function $f(x)$ could be represented in the form

$$
f(x)=\frac{a_{0}}{2}+\sum_{n=1}^{\infty}\left(a_{n} \cos n x+b_{n} \sin n x\right)
$$

with $a_{n}$ and $b_{n}$ given by the integrals

$$
\begin{aligned}
& a_{n}=\frac{1}{\pi} \int_{-\pi}^{\pi} f(x) \cos n x d x, n=0,1,2, \ldots \\
& b_{n}=\frac{1}{\pi} \int_{-\pi}^{\pi} f(x) \sin n x d x, n=1,2, \ldots .
\end{aligned}
$$

The trigonometric series given above is called the Fourier series corresponding to $f(x)$ and the constants $a_{n}$ and $b_{n}$ are called the Fourier coefficients of the function.

We should point out, however, some trigonometric series are not Fourier series. A trigonometric series of the form

$$
\frac{a_{0}}{2}+\sum_{n=1}^{\infty}\left(a_{n} \cos n x+b_{n} \sin n x\right)
$$


is not called a Fourier series unless there is some function $f(x)$ from which the coefficients in the series may be calculated by the formulas given above for $a_{n}$ and $b_{n}$.

The integrals defining $a_{n}$ and $b_{n}$ must be meaningful, i.e., the integrals exist either as proper Riemann integrals, Lebesgue integrals or as improper integrals. Throughout this Report when the Fourier series of a function is being studied the function will be assumed Riemann integrable. Most of the functions arising in ordinary applications are bounded and are continuous except possibly at a finite number of points and are therefore integrable in the sense of Riemann. Fourier series are useful in dealing with periodic functions but if the function is not periodic, it cannot be represented by a Pourier series. There is, however, an expression involving improper integrals instead of infinite series, which under suitable conditions gives a valid representation of a nonperiodic function. Then we say the function is represented by its Fourier integral.

The purpose of this Report is to acquaint the reader with some of the most important aspects of the theory and applications of Fourier series and Fourier integrals. 


\section{Fourier Series of a Function}

Definition 1.1. If $f(x)$ is a function which is defined on the interval $a \leq x \leq b$ and such that the integrals

$$
\begin{aligned}
& a_{n}=1 / \pi \int_{-n}^{\pi} f(x) \cos n x d x, \quad n=0,1,2, \ldots \\
& b_{n}=1 / \pi \int_{-n}^{\pi} f(x) \text { sin } n x d x, \quad n=1,2, \ldots
\end{aligned}
$$

have a meaning, we form the series

$$
\frac{a_{0}}{2}+\sum_{n=1}^{\infty}\left(a_{n} \cos n x+b_{n} \sin n x\right)
$$

and call this the Fourier series corresponding to $f(x)$.

The function determines its Fourier series quite apart from any consideration as to whether the series converges or, if it does converge, whether or not its sum is $f(x)$. If the Fourier series of a function $f(x)$ is formed without deciding in advance whether it converges to $f(x)$, then we write

$$
f(x) \sim \frac{a_{0}}{2}+\sum_{n=1}^{\infty}\left(a_{n} \cos n x+b_{n} \sin n x\right) .
$$

This notation means merely that the Fourier series on the right corresponds to the function $f(x)$. The sign $\sim$ can be replaced by the sign $=$ only if we succeed in proving that the series converges and that its sum equals $f(x)$.

Before we find the Fourier series corresponding to a particular function, we should give an explanation of how we arrived at the integrals for the Fourier coefficients $a_{n}$ and $b_{n}$ given by (1) and (2). First consider the orthogonality of sine and cosine functions. For 
any integer $n>0$

(3)

$$
\int_{-\pi}^{\pi} \cos n x d x=0
$$

(4)

$$
\int_{-\pi}^{\pi} \sin n x d x=0
$$

(5)

$$
\int_{-\pi}^{\pi} \cos ^{2} n x d x=\pi
$$

(6)

$$
\int_{-\pi}^{\pi} \sin ^{2} n x d x=\pi \text {. }
$$

Let $m$ and $n$ denote positive integers. Then

$$
\int_{-\pi}^{\pi} \cos n x \cos m x d x=0 \text { if } m+n \text {. }
$$

$$
\int_{-\pi}^{\pi} \sin n x \sin m x d x=0 \text { if } m
$$

$$
\int_{-\pi}^{\pi} \sin n x \cos m x d x=0 \text { for any } m \text { and } n \text {. }
$$

Suppose the function $f(x)$ of period $2 \pi$ has the expansion

$$
f(x)=\frac{a_{0}}{2}+\sum_{k=1}^{\infty}\left(a_{k} \cos k x+b_{k} \sin k x\right) .
$$

It will be assumed that the series (10) and the series to be written later can be integrated term by term. Integrating (10) from $-\boldsymbol{n}$ to $\boldsymbol{\pi}$, we obtain 


$$
\int_{-\pi}^{\pi} f(x) d x=\int_{-\pi}^{\pi} d x+\sum_{k=1}^{\infty}\left(a_{k} \int_{-\pi}^{\pi} \cos k x d x+b_{k} \int_{-\pi}^{\pi} \sin k x d x\right) .
$$

By (3) and (4) all the integrals in the sum vanish, so that

$$
\int_{-\pi}^{\pi} f(x) d x=\pi a_{0}
$$

Next, multiply both sides of (10) by cox $\mathrm{nx}$ and integrate the result from $-\pi$ to $\boldsymbol{n}$, as before, obtaining

$$
\begin{aligned}
& \int_{-\pi}^{\pi} f(x) \cos n x d x=\frac{a_{0}}{2} \int_{-\pi}^{\pi} \cos n x d x+\sum_{k=1}^{\infty}\left(a_{k} \int_{-\pi}^{\pi} \cos k x \cos n x d x\right. \\
& \left.+b_{k} \int_{-\pi}^{\pi} \sin k x \cos n x d x\right)
\end{aligned}
$$

By (3) the first integral on the right vanishes. By (7) and (9) all the integrals in the sum vanish except one. Thus (11) reduces to

$$
\int_{-\pi}^{\pi} f(x) \cos n x d x=a_{n} \int_{-\pi}^{\pi} \cos ^{2} n x d x .
$$

Now by (5),

$$
a_{n}=\frac{1}{\pi} \int_{-\pi}^{\pi} f(x) \cos n x d x, \quad n=0,1,2, \ldots
$$

Similarly,

$$
b_{n}=\frac{1}{\pi} \int_{-\pi}^{\pi} f(x) \sin n x d x, \quad n=1,2, \ldots .
$$


Before computing the Fourier series corresponding to a particular function $f(x)$, there is a consideration which will sometimes simplify the calculation of the Fourier coefficients.

Definition 1.2. The function $f(x)$ is said to be an even function if $f(x)=f(-x)$ for every $x$.

This definition implies that the graph of any even function is symmetric with respect to the $y$-axis. It follows from the interpretation of the integral as an area that for even functions defined and integrable on the interval $[-\pi, n]$,

$$
\int_{-\pi}^{n} f(x) d x=2 \int_{0}^{n} f(x) d x .
$$

Definition 1.3. The function $f(x)$ is said to be odd if $f(-x)=-f(x)$ for every $x$.

The graph of any odd function $f(x)$ is symmetric with respect to the origin. Thus, for odd functions defined and integrable on the interval $[-\pi, n]$,

$$
\int_{-\pi}^{n} f(x) d x=0
$$

The following properties are consequences of the definition of even and odd functions:

(i) The product of two even or two odd function is an even function.

(ii) The product of an even and an odd function is an odd function. 
Thus if $\phi(x)$ and $\psi(x)$ are even functions, then for $f(x)=\phi(x) \psi(x)$, we have

$$
f(-x)=\phi(-x) \psi(-x)=\phi(x) \psi(x)=f(x),
$$

while if $\phi(x)$ and $\psi(x)$ are odd,

$$
f(-x)=\phi(-x) \psi(-x)=[-\phi(x)] \quad[-\psi(x)]=\phi(x) \psi(x)=f(x) .
$$

This proves Property (i). On the other hand, if $\phi(x)$ is even and $\psi(x)$ is odd, then

$$
f(-x)=\phi(-x) \psi(-x)=\phi(x)[-\psi(x)]=-\phi(x) \psi(x)=-f(x)
$$

which proves Property (ii).

Let $f(x)$ be an even function defined on the interval $[-n, n]$. Since $\cos n x(n=0,1,2, \ldots)$ is an even function, then by Property (i) the function $f(x) \cos n x$ is also even. On the other hand, the function $\sin n x(n=1,2, \ldots)$ is odd, so that the function $f(x)$ sin $\mathrm{nx}$ is also odd, by Property (ii). Then, using (12), (13), (14) and (15), we find that the Fourier coefficients of the even function $f(x)$ are

$$
\begin{aligned}
& a_{n}=\frac{1}{n} \int_{-\pi}^{\pi} f(x) \cos n x d x=\frac{2}{\pi} \int_{0}^{\pi} f(x) \cos n x d x, n=0,1,2 \ldots \\
& b_{n}=\frac{1}{\pi} \int_{-\pi}^{n} f(x) \sin n x d x=0, \quad n=1,2, \ldots .
\end{aligned}
$$

Therefore, the Fourier series of an even function contains only cosines, i.e.,

$$
f(x) \sim \frac{a_{0}}{2}+\sum_{n=1}^{\infty} a_{n} \cos n x .
$$


The series above is known as the Fourier cosine series. Now let $f(x)$ be an odd function defined on the interval $[-\pi, \pi]$. Since $\cos n x(n=0,1,2, \ldots)$ is an even function, then by Property (ii) $f(x) \cos n x$ is an odd function. On the other hand, the function $\sin n x(n=1,2, \ldots)$ is odd and by Property (i) the function $f(x)$ sin $n x$ $\geq$ is even. Then, using (12), (13), (14) and (15) we find that the Fourier coefficients of the odd function $f(x)$ are

$$
\begin{aligned}
& a_{n}=\frac{1}{\pi} \int_{-\pi}^{\pi} f(x) \cos n x d x=0, \quad n=0,1,2, \ldots \\
& b_{n}=\frac{1}{\pi} \int_{-n}^{\pi} f(x) \sin n x d x=\frac{2}{\pi} \int_{0}^{\pi} f(x) \sin n x d x, n=1,2, \ldots
\end{aligned}
$$

Therefore, the Fourier series of an odd function contains only sines, i.e.,

$$
f(x) \sim \sum_{n=1}^{\infty} b_{n} \sin n x .
$$

This series becomes the Fourier since series.

The following example illustrates the procedure for finding the Fourier series corresponding to a particular function.

Example: Find the Fourier series corresponding to the function $f(x)=x^{2} / 4$ when $-\pi \leq x \leq \pi$.

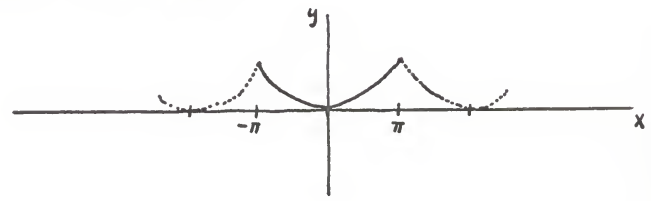


The function is even, so $b_{n}=0$ and

For $\mathrm{n}=0$

$$
a_{n}=\frac{1}{\pi} \int_{-\pi}^{n} \frac{x^{2}}{4} \cos n x d x .
$$

$$
a_{0}=\frac{1}{\pi} \int_{-\pi}^{\pi} \frac{x^{2}}{4} d x=\frac{\pi^{2}}{6} .
$$

If $n>0$

$$
\begin{aligned}
a_{n} & =\frac{1}{\pi} \int_{-\pi}^{\pi} \frac{x^{2}}{4} \cos n x d x \\
& =\frac{1}{2 \pi} \int_{0}^{\pi} x^{2} \cos n x d x \\
& =\frac{1}{2 \pi}\left[\frac{x^{2}}{n} \sin n x-\frac{2}{n^{3}} \sin n x+\frac{2 x}{n^{2}} \cos n x\right]_{0}^{\pi} \\
& =\frac{1}{n^{2}} \cos n \pi=\frac{1}{n^{2}}(-1)^{n} .
\end{aligned}
$$

Thus,

$$
f(x) \sim \frac{\pi^{2}}{12}-\frac{\cos x}{1^{2}}+\frac{\cos 2 x}{2^{2}}-\frac{\cos 3 x}{3^{2}}+\cdots \cdot
$$

Notice that we write $\sim$ rather than $=$, for as yet we have not shown that the series converges to the value $f(x)$.

If it is required to expand a function $f(x)$ of period $2 L$ in a Fourier series, set $x=L t / n$, thereby obtaining the function $\phi(t)=$ $f(L t / \pi)$ of period $2 \pi$. 
For $\phi(t)$ form the Fourier series

$$
\phi(t) \sim \frac{a_{0}}{2}+\sum_{n=1}^{\infty}\left(a_{n} \cos n t+b_{n} \sin n t\right),
$$

where

$$
\begin{aligned}
& a_{n}=\frac{1}{\pi} \int_{-\pi}^{n} \phi(t) \cos n t d t=\frac{1}{\pi} \int_{-\pi}^{\pi} f(L t / \pi) \cos n t d t, n=0,1,2, \ldots \\
& b_{n}=\frac{1}{\pi} \int_{-\pi}^{\pi} \phi(t) \sin n t d t=\frac{1}{\pi} \int_{-\pi}^{\pi} f(L t / \pi) \sin n t d t, n=1,2, \ldots .
\end{aligned}
$$

Returning to the original variable $x$ by setting $t=\pi x / L$, we obtain

$$
f(x) \sim \frac{a_{0}}{2}+\sum_{n=1}^{\infty}\left(a_{n} \cos \frac{\pi n x}{L}+b_{n} \sin \frac{\pi n x}{L}\right)
$$

where

$$
\begin{aligned}
& a_{n}=\frac{1}{L} \int_{-L}^{L} f(x) \cos \frac{\pi n x}{L} d x, n=0,1,2, \ldots \\
& b_{n}=\frac{1}{L} \int_{-L}^{L} f(x) \sin \frac{\pi n x}{L} d x, n=1,2, \ldots .
\end{aligned}
$$

The coefficients are the Fourier coefficients of $f(x)$ and the series is the Fourier series of $f(x)$ over the general interval $[-L, L]$.

\section{Convergence of Fourier Series}

In order to establish conditions under which a Fourier series converges to its function, we introduce a few preliminary therorems on limits of trigonometric integrals. The proofs of these theorems will be omitted. The integrals involved in these theorems are known 
as Dirichlet's integrals.

Definition 2.1. Any integral of the form

$$
\int_{0}^{a} \phi(t) \frac{\sin k t}{\sin t} d t \quad \text { or } \int_{0}^{a} \phi(t) \frac{\sin k t}{t} d t
$$

is called a Dirichlet integral.

Definition 2.2. A function is sectionally continuous in a finite interval if that interval can be subdivided into a finite number of intervals in each of which the function is continuous and has finite limits as the variable approaches either end point from the interior.

Theorem 2.1. If $f(x)$ is sectiona1ly continuous in the interval $a \leq x \leq b$, then

$$
\lim _{k \rightarrow \infty} \int_{a}^{b} f(x) \sin k x d x=0 .
$$

Definition 2.3. The symbol $f\left(x_{0}+0\right)$ denotes the 1 imit of $f(x)$ as $x$ approaches $x_{0}$ from the right. The symbol $f\left(x_{0}-0\right)$ denotes the limit of $f(x)$ as $x$ approaches $x_{0}$ from the left.

Definition 2.4. The right-hand derivative of $f(x)$ at $x_{0}$ is given by the following limit:

$$
\lim _{h \rightarrow 0} \frac{F\left(x_{0}+h\right)-F\left(x_{0}+0\right)}{h}
$$

The left-hand derivative of $f(x)$ at $x_{0}$ is

$$
\lim _{h \rightarrow 0} \frac{F\left(x_{0}-0\right)}{h}-F\left(x_{0}-h\right)
$$

where $h$ is ppsitive. 
Theorem 2.2. If $f(x)$ is sectionally continuous in the interval $0 \leq x \leq b$ and has a right-hand derivative at $x=0$, then

$$
\lim _{k \rightarrow \infty} \int_{0}^{b} f(x) \frac{\sin k x}{x} d x=\pi / 2 f(+0) \text {. }
$$

Theorem 2.3. If $f(x)$ is sectionally continuous in the interval $(a, b)$ and has derivatives from the right and left at a point $x=x_{0}$, where $a<x_{0}<b$, then

$$
\lim _{x \rightarrow \infty} \int_{a}^{b} f(x) \frac{\sin k\left(x-x_{0}\right)}{x-x_{0}} d x=\pi / 2\left[f\left(x_{0}+0\right)+f\left(x_{0}-0\right)\right] .
$$

The following theorem gives conditions under which a Fourier series corresponding to a function converges to that function. Such a theorem is called a Fourier theorem.

Theorem 2.4. Let $f(x)$ satisfy the conditions: (a) $f(x+2 \pi)=f(x)$ for all values of $x$, and (b) $f(x)$ is sectionally continuous in the interval $(-\pi, \pi)$. Then the Fourier series

$$
\frac{1}{2} a_{0}+\sum_{n=1}^{\infty}\left(a_{n} \cos n x+b_{n} \sin n x\right)
$$

where

$$
a_{n}=\frac{1}{\pi} \int_{-\pi}^{\pi} f(x) \cos n x d x, n=0,1,2, \ldots
$$

$$
b n=\frac{1}{\pi} \quad \int_{-n}^{n} f(x) \sin n x d x, n=1,2, \ldots
$$


converges to the value

$$
\frac{1}{2}[f(x+0)+f(x-0)]
$$

at every point where $f(x)$ has a right-and left-hand derivative.

Proof: The series (1) with coefficients (2) can be put in the form

$$
\frac{1}{2 \pi}=\int_{-\pi}^{n} f(t) d t+1 / \pi \sum_{m=1}^{\infty}\left(\int_{-\pi}^{\pi} f(t) \cos [m(t-x)] d t\right) .
$$

The sum $S_{n}(x)$ of the first $n+1$ terms of the series can therefore be written

$$
s_{n}(x) \quad \frac{1}{\pi} \int_{-\pi}^{\pi} f(t)\left\{\frac{1}{2}+\sum_{m=1}^{n} \cos [m(t-x)]\right\} d t .
$$

Applying Lagrange's trigonometric identify

$$
2 \sum_{n=1}^{N} \cos n \theta=-1 \frac{\sin (N+1 / 2) \theta}{\sin 1 / 2 \theta}
$$

to the sum of cosines here, we have

$$
\sin (x)=\frac{1}{\pi} \int_{-\pi}^{\pi} f(t) \frac{\sin [(n+1 / 2)(t-x)]}{2 \sin [1 / 2(t-x)]} d t .
$$

The integrand here is a periodic function of $t$ with period $2 \pi$; hence its integral over every interval of length $2 \pi$ is the same. Let us integrate over the interval $(a, a+2 \pi)$, where the number a has been selected so that the point $x$ is in the interior of that interval: that is, $a<x<a+2 \pi$. 
Introducing the factor $(t-x)$ in both the numerator and the denominator of the integrand, we have

$$
s_{n}(x)=1 / \pi \int_{a}^{a+2 n} F(t) \frac{\sin [(n+1 / 2)(t-x)]}{(t-x)} d t
$$

where

$$
F(t)=f(t) \frac{(t-x)}{2 \sin [1 / 2(t-x)]} .
$$

Now

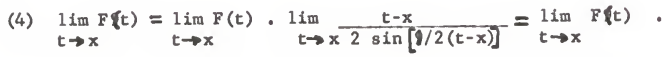

Moreover, $F(t)$ is written as the product of two functions each of which is sectionally continuous in every interval and has a derivative from the right and left at the point $t=x$. This was assumed in the theorem for the first factor $f(t)$ and it is easily verified for the second. Therefore, $F(t)$ is sectionally continuous, and its derivatives from the right and left exist at $t=x$.

Therefore $F(t)$ satisfies the conditions of Theorem 2.3 in which $x_{0}=x$ and $k=n+1 / 2$. Using Theorem 2.3 for the integral in equation (3), we have

$$
\lim _{n \rightarrow \infty} S_{n}(x)=1 / 2[F(x+0)+F(x-0)] \text {. }
$$

But according to equation (4),

$$
F(x+0)=f(x+0), \quad F(x-0)=f(x-0),
$$


and therefore

$$
\lim _{n \rightarrow \infty} S_{n}(x)=1 / 2[F(x+0)+F(x-0)] \text {. }
$$

This completes the proof of the theorem.

Differentiation and Integration of Fourier Series

The following theorem gives a complete set of sufficiency conditions for the termwise differentiation of a Fourier series.

Theorem 3.1. Let $f(x)$ be a continuous function in the interval $-\pi \leq x \leq \pi$ such that $f(\pi)=f(-\pi)$, and let its derivative $f^{\prime}(x)$ be sectionally continuous in that interval. Then the one-sided derivatives of $f(x)$ exist, and $f(x)$ is represented by its Fourier series

(1) $f(x)=1 / 2 a_{0}+\sum_{n=1}^{\infty}\left(a_{n} \cos n x+b_{n} \sin n x\right), \quad-n \leq x \leq \pi$ where

$$
a_{n}=1 / \pi \int_{-\pi}^{\pi} f(x) \cos n x d x
$$

$$
b_{n}=1 / \pi \int_{-\pi}^{\pi} f(x) \sin n x d x
$$

and at each point where $f^{\prime}(x)$ has a derivative that series can be differentiated termise; that is, (3) $f^{\prime}(x)=\sum_{n=1}^{\infty} n\left(-a_{n} \sin n x+b_{n} \cos n x\right),-\pi<x<\pi$. 
Proof: Since $f^{\prime}(x)$ satisfies the conditions of Theorem 2.4 , it is represented by its Fourier series at each point where its derivative $f^{\prime \prime}(x)$ exists. At such a point $f^{\prime}(x)$ is continuous, so that

(4) $f^{\prime}(x)=1 / 2 a_{0}^{\prime}+\sum_{n=1}^{\infty}\left(a_{n}^{\prime} \cos n x+b_{n}^{\prime} \sin n x\right)$,

where

$$
\begin{aligned}
& a_{n}^{\prime}=1 / \pi \int_{-\pi}^{\pi} f^{\prime}(x) \cos n x d x \\
& b_{n}^{\prime}=1 / \pi \int_{-\pi}^{\pi} f^{\prime}(x) \sin n x d x .
\end{aligned}
$$

These integrals can be integrated by parts, since $f(x)$ is continuous and $f^{\prime}(x)$ is sectionally continuous. Therefore

$$
\begin{aligned}
a_{n}^{\prime} & =\frac{1}{\pi}\left[\left.f(x) \cos n x\right|_{-\pi} ^{\pi}+n \int_{-\pi}^{\pi} f(x) \sin n x d x\right\} \\
& =\frac{1}{\pi}[f(x) \cos n x]_{-\pi}^{\pi}+n b_{n} \\
& =\frac{1}{\pi}[f(\pi) \cos n \pi-f(-\pi) \cos (-n \pi)]+n b_{n} \\
& =\frac{1}{\pi} \cos n n[f(\pi)-f(-n)]+n b_{n} \cdot
\end{aligned}
$$

This reduces to $n b_{n}$ because $f(\pi)=f(-\pi)$. Furthermore, $a_{0}^{\prime}=0$. Similarly,

$$
b_{n}^{\prime}=\frac{1}{\pi}[f(x) \sin n x]_{-\pi}^{\pi}-\frac{n}{\pi} \int_{-\pi}^{\pi} F(x) \cos n x d x=-n a_{n} .
$$


Substituting these values of $a_{n}^{\prime}$ and $b_{n}^{\prime}$ into equation (4), we have

$$
f^{\prime}(x)=\sum_{n=1}^{\infty}\left(n b_{n} \cos n x-n a_{n} \sin n x\right)
$$

This is the equation (3) which was obtained by differentiating (1) term by term; hence the theorem is proved.

Termwise integration of a Fourier series is posstble under conditions much more general than those for differentiation. It will be shown in the following theorem that it is not even essential that the original series converge to its function, in order that the integrated series converge to the integral of the function.

Theorem 3.2. Let $f(x)$ be sectionally continuous in the interval $(-\boldsymbol{\pi}, \pi)$. Then whether the Fourier series corresponding to $f(x)$,

(5) $f(x) \sim 1 / 2 a_{0}+\sum_{n=1}^{\infty}\left(a_{n} \cos n x+b_{n} \sin n x\right)$,

converges or not, the following equality is true:

$$
\int_{-\pi}^{x} f(x) d x=a_{0} / 2(x+\pi)+\sum_{n=1}^{\infty} \frac{1}{n}\left[a_{n} \sin n x-b_{n}(\cos n x-\cos n)\right],
$$

when $-\pi \leq x \leq \pi$. The latter series is obtained by integrating the former one term by term.

Proof: Since $f(x)$ is sectionally continuous, the function $F(x)$, where

$$
F(x)=\int_{-\pi}^{x} f(t) d t-1 / 2 a_{0} x
$$


is continuous; moreover

$$
F^{\prime}(x)=f(x)-1 / 2 a_{0} \text {, }
$$

except at points where $f(x)$ is discontinuous, and even there $F(x)$ has right-and left-hand derivatives. Also,

$$
F(\pi)=\int_{-\pi}^{\pi} f(x) d x-1 / 2 a_{0} \pi=a_{0} \pi-1 / 2 a_{0} \pi=1 / 2 a_{0} \pi
$$

and

$$
F(-\pi)=1 / 2 a_{0} ;
$$

hence

$$
F(\pi)=F(-\pi)
$$

According to Theorem 2.4 then, for all $x$ in the interval $-\nabla \leq x \leq \pi$, it is true that

$$
F(x)=1 / 2 A_{0}+\sum_{n=1}^{\infty}\left(A_{n} \cos n x+B_{n} \sin n x\right)
$$

where

$$
\begin{aligned}
& A_{n}=1 / \pi \int_{-\pi}^{\pi} F(x) \cos n x d x \\
& B_{n}=1 / \pi \int_{-\pi}^{\pi} F(x) \sin n x d x .
\end{aligned}
$$

Since $F(x)$ is continuous and $F^{\prime}(x)$ is sectionally continuous, the integrals for $A_{n}$ and $B_{n}$ can be integrated by parts. Thus if $n \neq 0$, 


$$
\begin{aligned}
A_{n} & =1 / \pi[1 / n F(x) \sin n x]_{-\pi}^{\pi}-\frac{1}{n \pi} \int_{-\pi}^{\pi} F^{\prime}(x) \sin n x d x \\
& =-1 / n \pi \int_{-\pi}^{\pi}\left[f(x)-a_{0} / 2\right] \sin n x d x \\
& =\frac{-1}{n \pi} \int_{-\pi}^{\pi} f(x) \sin n x d x+a_{0} / 2 n \pi \int_{-\pi}^{\pi} \sin n x d x \\
& =-1 / n \text {. }
\end{aligned}
$$

Similarly,

$$
B_{n}=a_{n} / n \text {; }
$$

hence

$$
F(x)=1 / 2 A_{0}+\sum_{n=1}^{\infty} 1 / n\left(a_{n} \sin n x-b_{n} \cos n x\right) .
$$

But since $F(\pi)=(1 / 2) a_{0} \pi$,

$$
1 / 2 a_{0} \pi=1 / 2 A_{0}-\sum_{n=1}^{\infty} 1 / n b_{n} \cos n \pi .
$$

Substituting the value of $A_{0}$ given here in equation (8),

$$
\begin{aligned}
F(x) & =1 / 2 a_{0} \pi+\sum_{n=1}^{\infty} 1 / n b_{n} \cos n \pi+\sum_{n=1}^{\infty} 1 / n\left(a_{n} \sin n x-b_{n} \operatorname{cox} n x\right) \\
& =1 / 2 a_{0} \pi+\sum_{n=1}^{\infty} 1 / n\left(a_{n} \sin n x+b_{n} \cos n \pi-b_{n} \cos n x\right) \\
& =1 / 2 a_{0} \pi+\sum_{n=1}^{\infty} 1 / n\left[a_{n} \sin n x-b_{n}(\cos n x-\cos n \pi)\right] .
\end{aligned}
$$


In view of equation (7)

$$
\begin{aligned}
F(x) & =\int_{-n}^{x} f(x) d x-1 / 2 a_{0} x \\
& =1 / 2 a_{0} \pi+\sum_{n=1}^{\infty} 1 / n\left[a_{n} \sin n x-b_{n}(\cos n x-\cos n n)\right]
\end{aligned}
$$

Then,

$$
\int_{-\pi}^{x} F(x) d x=1 / 2 a_{0}(x+\pi)+\sum_{n=1}^{\infty} 1 / n\left[a_{n} \sin n x-b_{n}(\cos n x-\cos n n)\right]
$$

and the theorem is proved.

\section{The Fourier Integral}

Fourier series are useful in dealing with periodic functions. If a function is defined for all real values of $x$, but is not periodic, it cannot be represented by a Fourier series. There is, however, a method involving improper integrals instead of infinite series, which under suitable conditions gives a valid representation of a function $f(x)$.

Definition 4.1. The expression

$$
1 / \pi \int_{0}^{\infty} d u \int_{-\infty}^{\infty} f(t) \cos [u(t-x)] d t
$$

is called the Fourier integral of $f(x)$.

The analogy between a Fourier integral and a Fourier series may be seen as follows: For any $u \geq 0$ let 
(2)

$$
\begin{aligned}
& a(u)=1 / \pi \int_{-\infty}^{\infty} F(t) \cos u t d t \\
& b(u)=1 / \pi \int_{-\infty}^{\infty} F(t) \sin u t d t .
\end{aligned}
$$

The functions $a(u)$ and $b(u)$ are analogous to the Fourier coefficients $a_{n}$ and $b_{n}$. Then form the integral

$$
\int_{0}^{\infty}[a(u) \cos u x+b(u) \sin u x] d u,
$$

which is analogous to the Fourier series (10).

Now,

$$
\begin{aligned}
a(u) \cos u x+b(u) \sin u x=1 / \pi & \int_{-\infty}^{\infty} f(t)[\cos u t \cos u x \\
& +\sin u t \sin u x] d t \\
& =1 / \pi \int_{-\infty}^{\infty} P(t) \cos [u(t-x)] d t .
\end{aligned}
$$

Thus, the integral (3) can be put in the form (1). All these cons1derations assume that the integrals in question are convergent.

The following results for the Fourier integral are analogous to those for the Fourier series of a function. If $f(x)$ is an even function, $f(t) \cos$ ut is even and $f(t)$ sin ut is odd, so that $b(u)=0$ and

$$
a(u)=2 / \pi \int_{0}^{\infty} f(t) \cos u t d t .
$$

In this case the Fourier Integral takes the form

$$
2 / \pi \int_{0}^{\infty} \cos u x d u \int_{0}^{\infty} f(t) \cos u t d t,
$$

which we call the Fourier cosine integral. For an odd function 
$f(x)$, we can show that $a(u)=0$ and

$$
b(u)=2 / \pi \int_{0}^{\infty} \sin u x d u \int_{0}^{\infty} f(t) \sin u t d t,
$$

we call this the Fourier sine integral.

The conditions given in the following theorem, called the

Fourier integral theorem, insure that $f(x)$ may be represented by its Fourier integral.

Theorem 4.1. Let $f(x)$ be sectionally continuous in every finite interval $(a, b)$, and let $\int_{-\infty}^{\infty}|f(x)| d x$ converge. Then at every point $x$, $(-\infty<x<\infty)$, where $f(x)$ has a right-and left-hand derivative, $f(x)$ is represented by its Fourier integral as follows:

(4) $1 / 2[F(x+0)+F(x-0)]=1 / \pi \int_{0}^{\infty} d \alpha \int_{-\infty}^{\infty} f(t) \cos [\alpha(t-x)] d t$.

Proof: In every interval $(a, b), f(x)$ satisfies the conditions of Theorem 2.3 , so that

(5) $\pi / 2[f(x+0)+f(x-0)]=\lim _{\alpha \rightarrow \infty} \int_{a}^{b} f(t) \frac{\sin [\alpha(t-x)]}{t-x} d t$ at any point $x(a<x<b)$, where $f(x)$ has a right-and left-hand derivative.

To prove (4) we have to show that if $\epsilon>0$,

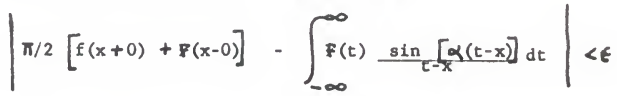

for all sufficiently large values of $\alpha$. 
Whenever $a<x$,

$$
\begin{aligned}
\int_{-\infty}^{a} f(t) \frac{\sin [\alpha(t-x)] d t}{t-x} \mid & \leq \int_{-\infty}^{a}\left|f(t) \frac{\sin [\alpha(t-x)]}{t-x}\right| d t \\
& \leq \int_{-\infty}^{a} \frac{|f(t)|}{|t-x|} d t \\
& \leq \int_{-\infty}^{-a} \frac{|f(t)|}{|a-x|} d t \\
& =\frac{1}{x-a} \int_{-\infty}^{\infty}|f(t)| d t \\
& \leq \frac{1}{x-a} \int_{-\infty}^{\infty} f(t) d t
\end{aligned}
$$

Since the integral $\int|f(x)| d x$ converges, we can replace $\int_{-\infty}|f(t)| d t$ by a constant, say $k$. Hence

$$
\left|\int_{-\infty}^{a} f(t) \frac{\sin [\alpha(t-x)]}{(t-x)} d t\right| \leqslant \frac{1}{x-a} \cdot k .
$$

Now choose a such that $a<x-3 k / t$ and the integral will be numerically less than $\in / 3$. Similarly b can be chosen large enough so that

$$
\left|\int_{b}^{\infty} f(t) \frac{\sin [\alpha(t-x)]}{(t-x)} d t\right|
$$

is less than $\epsilon / 3$.

$$
\text { Now split up the integral in (6) in the manner }
$$

$$
\int_{-\infty}^{\infty}=\int_{-\infty}^{a}+\int_{a}^{b}+\int_{b}^{\infty} .
$$


Hence (6) becomes

$$
\begin{aligned}
& \int \pi / 2[P(x+0)+F(x-0)]-\int_{-\infty}^{a} f(t) \frac{\sin [\alpha(t-x)]}{(t-x)} d t-\int_{a}^{b} f(t) \frac{\sin [\alpha(t-x)]}{(t-x)} d t \\
& -\int_{b}^{\infty} P(t) \frac{\sin [\alpha(t-x)]}{(t-x)} d t \mid \leq
\end{aligned}
$$

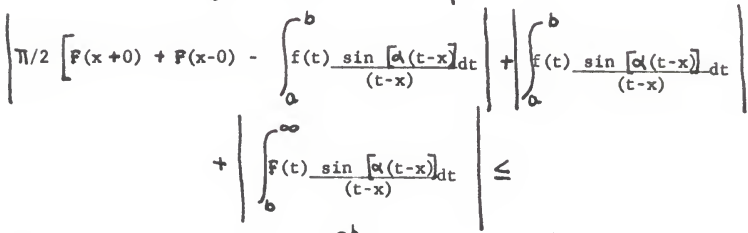

$$
\begin{aligned}
& \mid n / 2\left[(F(x+0)+F(x-0)]-\int_{a}^{b} F(t) \frac{\sin [\alpha(t-x)]}{(t-x)} d \tau \mid+2 \epsilon / 3\right. \text {. }
\end{aligned}
$$

The integral

$$
\int_{a}^{b} F(t) \frac{\sin [\alpha(t-x)]}{(t-x)} d t
$$

can be made to differ from the value $\pi / 2[f(x+0)+f(x-0)]$ by an amount numerically less than $f / 3$ by taking $\alpha$ sufficiently large, according to equation (5). Hence the integral

$$
\int_{-\infty}^{\infty} f(t) \frac{\sin [\alpha(t-x)]}{(t-x)} d t
$$

differs from the value $\pi / 2[f(x+0)+f(x-0)]$ by an amount less than E. That is, 
$\lim _{\alpha \rightarrow \infty} \int_{-\infty}^{\infty} F(t) \frac{\sin [\alpha(t-x)]}{(t-x)} d t=\pi / 2[f(x+0)+f(x-0)]$.

Writing the fraction in the integrand as an integral, and dividing by $\pi$, this becomes

$$
\begin{aligned}
1 / 2[f(x+0)+f(x-0)] & =\lim _{\alpha \rightarrow \infty} 1 / \pi \int_{-\infty}^{\infty} f(t) d t \int_{0}^{\alpha} \cos \left[\alpha^{\prime}(t-x)\right] d \alpha^{\prime} . \\
& =\lim _{\alpha \rightarrow \infty} 1 / n \int_{0}^{\alpha} d \alpha^{\prime} \int_{-\infty}^{\infty} f(t) \cos \left[\alpha^{\prime}(t-x)\right] d t .
\end{aligned}
$$

The inversion of order of integration in the last step is valid because the integrand does not exceed $|f(t)|$ in absolute value, so that the integra 1

$$
\int_{-\infty}^{\infty} f(t) \cos \left[\alpha^{\prime}(t-x)\right] d t
$$

converges uniformly for all $\alpha^{\prime}$. The last equation is the same as statement (4) in the theorem. Hence the theorem is proved.

Example. Show that the function $f(x)=0$ when $x<0, f(x)=e^{-x}$ when $x>0, f(0)=1 / 2$, is represented by its Fourier integral; hence show that the integral

$$
\int_{0}^{\infty} \frac{\cos x \alpha+\alpha \sin x \alpha}{1+\alpha^{2}} d \alpha
$$

has the value 0 if $x<0, \pi / 2$ if $x=0$ and $\pi e^{-x}$ if $x>0$.

The Fourier integral corresponding to an arbitrary function $f(x)$ is 
(2)

$$
\frac{1}{\pi} \int_{0}^{\infty} d \alpha \int_{-\infty}^{\infty} f(t) \cos [\alpha(t-x)] d t .
$$

For our problem,

$$
\int_{-\infty}^{\infty} f(t) \cos [\alpha(t-x)] d t=\int_{0}^{\infty} e^{-t} \cos [\alpha(t-x)] d t .
$$

Now integrating by parts, we have

$$
\int_{0}^{\infty} e^{-t} \cos [\alpha(t-x)] d t=\frac{\cos x \alpha+\alpha \sin x \alpha}{1+\alpha^{2}} .
$$

Thus, we have the integral

(3)

$$
\int_{0}^{\infty} \frac{\cos x \alpha+\alpha \sin x \alpha}{1+\alpha^{2}}
$$

to evaluate.

We can write the integral (3) as

(4)

$$
\int_{0}^{\infty} \frac{\cos x \alpha}{1+\alpha^{2}} d \alpha+\int_{0}^{\infty} \frac{\alpha \sin x \alpha}{1+\alpha^{2}} d \alpha .
$$

The above integrals cannot be integrated by any of the standard methods of integration used in elementary calculus. So we will use the residue theorem from complex variable theory.

Consider

$$
\int_{C} \frac{e^{i x z}}{1+e^{2}} d z
$$

where $C$ is the contour 
with $R>1$.

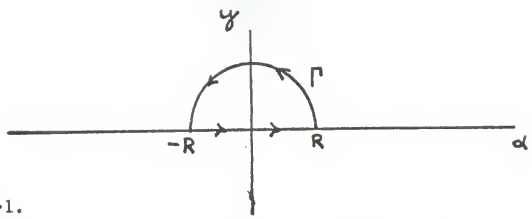

The integrand has simple poles at $z= \pm 1$, but only $z=1$ lies inside $C$. Then the residue at $z=1$ is

$$
\lim _{z \rightarrow l}\left\{(z-1) \frac{e^{i x z}}{(z-i)(z+1)}\right\}=\frac{e^{-x}}{2 i}
$$

The residue theorem states that the integral of a function $f(z)$ around $C$ if 2 i times the sum of the residues of $f(z)$ at the singularities enclosed by $C$.

Then,

$$
\oint_{C} \frac{e^{i x z^{2}}}{1+z^{2}}=2 \pi 1\left(\frac{e^{-x}}{2 l}\right)=\pi e^{-x}
$$

or,

$$
\begin{aligned}
& \int_{-R}^{R} \frac{e^{i x \alpha}}{1+\alpha^{2}} d \alpha+\int_{\Gamma} \frac{e^{i x z}}{1+z^{2}} d z=\pi e^{-x}, i . e ., \\
& \int_{-R}^{R} \frac{\cos x \alpha}{1+\alpha^{2}} d \alpha+i \int_{-R}^{R} \frac{\sin x \alpha}{1+\alpha^{2}}+\int_{\Gamma} \frac{e^{i x z}}{1+z^{2}} d z=\pi e^{-x} .
\end{aligned}
$$

The integrand of the integral on the left is an even function and the integrand of the second integral is an odd function. Hence, we have 
$2 \int_{0}^{R} \frac{\cos x \alpha}{1+\alpha^{2}} d \alpha+\int \frac{e^{i x z}}{1+z^{2}} d z=n e^{-x}$.

Next, take the limit of the integrals as $R \rightarrow \infty$. The integral around $\Gamma$ approaches zero. For, if $|F(z)| \leq M / R^{k}$ for $z=R^{i \theta}$, where $k>0, x>0$ and $M$ are constants, we can show that

$$
\begin{gathered}
\quad \lim _{R \rightarrow \infty} \int_{\Gamma=R e^{i \theta}} e^{i x z} F(z) d z=0 . \\
\int_{\Gamma} e^{i x \neq} F(z) d z=\int_{0}^{\pi} e^{i x e^{i \theta}} F\left(R e^{i \theta}\right) i R e^{i \theta} d \theta .
\end{gathered}
$$

Now,

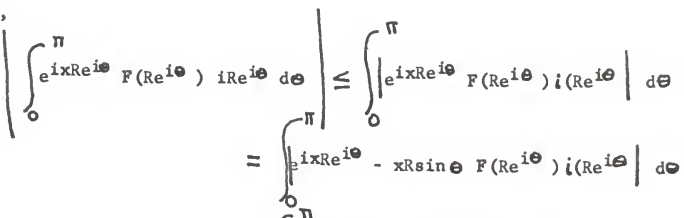

$$
\begin{aligned}
& =\int_{0}^{\pi} e^{-x \sin \theta}\left|F\left(R e^{i \theta}\right)\right| R d \theta \\
& \leq \frac{M}{R^{k-1}} \quad \int_{0}^{\pi} e^{-x R \sin \theta} d \theta \\
& =\frac{2 M}{R^{k-1}} \int_{0}^{\pi / z} e^{-x R \sin \theta} d \theta .
\end{aligned}
$$


29

Now $\sin \theta \geq 2 \theta / \pi$ for $0 \leq \theta \leq \pi / 2$. Then the last integral is less than or equal to

$$
\frac{2 M}{R^{k-1}} \int_{0}^{\pi / 2} e^{-\operatorname{axR} \theta / \pi} d \theta=\frac{\pi M}{x R^{k}} \quad\left(1-e^{-x R}\right) .
$$

As $R \rightarrow \infty$ this approaches zero, since $M$ and $k$ are positive. Thus (5) becomes

$$
2 \int_{0}^{\infty} \frac{\cos x \alpha}{1+\alpha^{2}} d \alpha=\pi e^{-x}
$$

or,

$$
\begin{aligned}
& \int_{0}^{\infty} \frac{\cos x \alpha}{1+\alpha 2} d \alpha=\pi / 2 \mathrm{e}^{-x}, \text { if } x>0 \\
& \text { Similarly, } \\
& \qquad \int_{0}^{\infty} \frac{\cos x \alpha}{1+\alpha^{2}} d \alpha=\pi / 2 \mathrm{e}^{x}, \text { if } x<0 \\
& \text { Next, let } F(x)=\int_{0}^{\infty} \frac{\cos x \alpha}{1+\alpha^{2}} d \alpha .
\end{aligned}
$$

Then,

$$
F^{\prime}(x)=-\int_{0}^{\infty} \frac{\sin x \alpha}{1+\alpha^{2}} d \alpha d
$$


Hence

$$
\begin{aligned}
\int_{0}^{\infty} \frac{\alpha \sin x \alpha}{1+\alpha^{2}} d \alpha & =\pi / 2 \mathrm{e}^{-x}, \text { if } x>0 \\
& =-\pi / 2 \mathrm{e}^{x}, \text { if } x<0
\end{aligned}
$$

Therefore,

$$
\begin{aligned}
\int_{0}^{\infty} \frac{\cos x \alpha+\alpha \sin x \alpha}{1+\alpha^{2}} d \alpha & =\pi e^{-x}, \text { if } x>0 \\
& =0, \text { if } x<0
\end{aligned}
$$

If $x=0$

$$
\int_{0}^{\infty} \frac{\cos x \alpha+\alpha \sin x \alpha}{1+\alpha^{2}} d \alpha=\pi / 2
$$

Application of Fourier Series and Integrals

In this section we shall discuss how Fourier series and integrals may enter into the solution of certain boundary value problems. First we will consider the vibrating string problem.

The equation of motion of the vibrating string fastened at both ends is

$$
\frac{\partial^{2} y}{\partial t^{2}}=a^{2} \quad \frac{\partial^{2} y}{\partial x^{2}} \text {. }
$$


Our problem is to find a solution $y(x, t)$ that satisfies the equation of motion and boundary conditions,

$$
y(0, t)=0, y(L, t)=0
$$

$$
\partial y(x, 0) / \partial t=0
$$

$$
y(x, 0)=f(x) .
$$

First we shall try to find solutions of (1) which satisfy the boundary conditions (2) and (3). We shall use the separation of variables method to find the solutions.

This method consists in looking for solutions of the form

$$
y(x, t)=X T
$$

where $X$ is a function of $X$ alone and $T$ is a function of $t$ alone. According to equation (5),

$$
\begin{aligned}
& \frac{\partial^{2} y}{\partial x^{2}}=T \frac{d^{2} x}{d x^{2}} \\
& \frac{\partial^{2} y}{\partial t^{2}}=x \frac{d^{2} T}{d t^{2}} .
\end{aligned}
$$

If these expressions are substituted into equation (1), we get

$$
\mathrm{x} \frac{\mathrm{d}^{2} \mathrm{~T}}{\mathrm{dt^{2 }}}=\mathrm{a}^{2} \mathrm{~T} \frac{\mathrm{d}^{2} \mathrm{X}}{\mathrm{dx^{2 }}}
$$

If we divide through by $a^{2} X T$, then equation (1) can be rewritten as 


$$
\frac{1}{a^{2} T} \frac{d^{2} T}{d t^{2}}=\frac{1}{x} \frac{d^{2} x}{d x^{2}}
$$

Since the member on the right is a function of $x$ alone, it cannot vary with $t$; it is equal to a function of $t$ alone, however, and thus it cannot vary with $x$. Hence both members must be equal to a constant, say $-w^{2}$, so that

$$
\begin{aligned}
& \frac{d^{2} x}{d x^{2}}=-w^{2} x \\
& \frac{d^{2} T}{d t^{2}}=-w^{2} a^{2} T,
\end{aligned}
$$

or

$$
\frac{d^{2} x}{d x^{2}}+w^{2} x=0
$$

$$
\frac{d^{2} T}{d t^{2}}+w^{2} a^{2} T=0
$$

It may not be obvious to the reader why we chose $-w^{2}$ instead of a constant B. Suppose that we had chosen a constant B so that

$$
\begin{aligned}
& X^{\prime \prime}-\beta x=0 \\
& T^{\prime \prime}-\beta a^{2} T=0 .
\end{aligned}
$$

The general solution of the first differential equation is

$$
x=M_{1} e^{x \sqrt{\beta}}+M_{2} e^{-x \sqrt{\beta}}
$$


where $M_{1}$ and $M_{2}$ are arbitrary constants. If $B$ is positive there are no values of $M_{1}$ and $M_{2}$ for which this function $X$ satisfies the conditions. Therefore, we choose the constant $-w^{2}$.

The general solution of equation (6) can be written as

$$
x=A \sin w x+B \cos w x,
$$

where $A$ and $B$ are arbitrary constants.

If $x(0)=0$, the constant $B$ must vanish. Then $A$ must be different from zero, since we are not interested in the trivial solution $x(x)=0$. So if $X(L)=0$, we must have

$$
\sin W L=0 .
$$

Hence there is a set of values of $w$, namely,

$$
w=n \pi / L
$$

for which the system consisting of equation (6) and boundary conditions (2) has solutions. These solutions are of the form

$$
X=A \sin n \pi x / L \text {. }
$$

Equation (7) has as its general solution

$$
T=C \sin \omega a t+D \cos w a t .
$$

If it is to satisfy condition $(3)$, then $T^{\prime}(0)=0$. Thus the coefficient $C$ must be zero, so we find that

$$
\mathrm{T}=\mathrm{Dcos} \text { wat. }
$$


Now if we substitute the value $n \pi / L$ for $w$, we have

$$
T=D \cos n \pi a t / L \text {. }
$$

Therefore all the functions

$$
A_{n} \sin n \pi x / L \cos n \pi a t / L, n=1,2, \ldots
$$

are solutions of our partial differential equation (1).

Any finite linear combination of the solutions will also be a

solution of (1) and satisfy conditions (2) and (3). When $t=0$, it will reduce to a linear combination of the functions

$$
\sin n \pi x / L \text {. }
$$

Thus condition (4) will not be satisfied unless the given function $f(x)$ can be written as a linear combination of sine functions.

Consider the infinite series,

$$
y=\sum_{n=1}^{\infty} A_{n} \sin (n \pi x / L) \cos (n \pi a t / L) .
$$

This series satisfies equation (1) provided it converges and it termwise differentiable; it also satisfies conditions (2) and (3). It will satisfy the nonhomogeneous condition (4) provided the numbers $A_{n}$ can be determined so that

$$
f(x)=\sum_{n=1}^{\infty} A_{n} \sin n \pi x / L \text {. }
$$

This series is the Fourier sine series for $f(x)$, so

$$
A n=1 / L \int_{-L}^{L} f(x) \sin n \pi x / L d x=2 / L \int_{0}^{L} F(x) \sin n \pi x / L d x .
$$


Thus the solution of equation (1) subject to the boundary conditions (2), (3) and (4) is

$$
y(x, t)=2 / L \sum_{n=1}^{\infty}\left(\sin n \pi x / L \cos n \pi a t / L \int_{0}^{L} F(x) \sin n \pi x / L d x\right) .
$$

From the nature of the physical problem, we expect that there should be just one solution $y(x, t)$ of equation (1) which satisfies the boundary conditions (2), (3) and (4), and this solution will represent the equation of motion of the string.

Another boundary value problem which we can solve with the aid of Fourier series is that of finding a formula for the temperatures in a slab of homogeneous material with the faces of the slab kept at temperature zero.

In this problem the temperature is a function of the variables $x$ and $t$. At each interior point this function $u(x, t)$ must satisfy the heat equation of one-dimensional flow,

$$
\frac{\partial u}{\partial t}=k \frac{d^{2} u}{d x^{2}}, 0<x<\pi, t>0
$$

It must also satisfy the boundary conditions

$$
u(+0, t)=0, u(\pi-0, t)=0
$$

$$
u(x,+0)=f(x)
$$

To find a particular solution of (1), we again will use the separayion of variables method. First we find a solution which satisfies conditions (2). Let $u=X(x) T(t)$. Hence, 


$$
\begin{aligned}
& \frac{\partial u}{\partial t}=X T^{\prime} \\
& \frac{\partial^{2} u}{\partial x^{2}}=T X^{\prime \prime} .
\end{aligned}
$$

Substituting these values for $\partial u / \partial t$ and $\partial^{2} u / \partial x^{2}$ in equation (1), we obtain

$$
\mathrm{XT}^{\prime}=\mathrm{kTX}
$$

or,

$$
\mathrm{X}^{\prime \prime} / \mathrm{X}=\mathrm{T}^{\prime} / \mathrm{kT} \text {. }
$$

The function on the left is a function of $\mathrm{x}$ alone, and the function on the right is a function of $t$, hence they must both equal a constant $\alpha$. Then,

(4)

$$
\begin{aligned}
& x^{\prime \prime}-\alpha x=0 \\
& T^{\prime}-\alpha k T=0 .
\end{aligned}
$$

If the function XT is to satisfy conditions (2), then

$$
\begin{aligned}
& x(0)=0 \\
& x(\pi)=0 .
\end{aligned}
$$


The solution of the first of the differential equations (4) is

$$
x=c_{1} e^{-\sqrt{\alpha} x}+c_{2} e^{\sqrt{\alpha x}}
$$

The solution of the first of equations (4) which satisfies the condition $x(0)=0$ is

$$
x=C^{\prime} \sinh \sqrt{\alpha} x \text {. }
$$

This can satisfy the condition $X(\pi)=0$ only if

$$
\alpha=-n^{2}, n=1,2, \ldots .
$$

Hence,

$$
x=C^{\prime} \sinh [ \pm(i n x)]
$$

or,

$$
x=C " \sin n x \text {. }
$$

The solution of the second of equations (4) is

$$
T=D_{1} e^{\alpha k t}
$$

or,

$$
\mathrm{T}=\mathrm{D}_{1} \mathrm{e}^{-\mathrm{n}^{2}} \mathrm{kt}
$$

Thus the solutions of equations (1) and (2) of the form $u=X T$ are

$$
b_{n} e^{-n^{2}} k t_{\sin } n x, n=1,2, \ldots
$$

where the constants $b_{n}$ are arbitrary. 
The infinite series of the functions (6) must also satisfy the nonhomogeneous condition (3). That is, the infinite series

$$
\sum_{n=1}^{\infty} b_{n} e^{-n^{2} k t} \sin n x,
$$

must reduce to $f(x)$ in $(0, \pi)$ when $t=0$. This occurs provided the coefficients $b_{n}$ are those of the Fourier sine series for $f(x)$; that is

$$
b_{n}=2 / \pi \quad \int_{0}^{\pi} f(x) \sin n x d x, n=1,2, \ldots .
$$

Hence the solution of the equations (1), (2) and (3) is

$$
u(x, t)=2 / \pi \sum_{n=1}^{\infty} e^{-n 2 k t} \sin n x \int_{0}^{\pi} f(x) \sin n x d x,
$$

provided this series converges to a function $u(x, t)$ such that

$u(x,+0)=u(x, 0)$ when $0<x<\pi, u(+0, t)=u(0, t)$ and $u(\pi-0, t)=$ $u(\pi, t)$, and provided the series can be differentiated termwise once with respect to $t$ and twice with respect to $x$ when $t>0$ and $0<x<\pi$

There are other boundary value problems whose solutions can be obtained with the aid of Fourier series. We will mention just a few of these problems: Variations of the problem of temperatures in a slab, temperatures in a sphere, steady temperatures in a rectangular plate and variations of the vibrating string problem. Another problem, which probably arises more often than any of the above is that of finding the motion of the damped harmonic oscillator subject to a periodic driving force. This problem can be solved with the aid of Fourier series, but there are other methods of solution which are more adaptable 
and are easier to use.

So far we have considered the solutions of boundary value problems with the use of Fourier series. Now we turn our attention to the solution of the following problem with the ald of the Fourier integral. Electricity is flowing in a thin plane sheet of infinite extent and the value of the potential function is given for every point in some straight line in the sheet. We will take the straight line as the $x$-axis and consider only those points for which $y$ is positive. Our problem is to find a function $V$ which will enable us to get the value of the potential at any point of the plane.

We have, then to satisfy the equation

$$
d^{2} v / d x^{2}+d^{2} v / d y^{2}=0
$$

subject to the conditions

$$
v=0 \text { when } y=\infty
$$

$$
v=f(x) \text { when } y=0 \text {, }
$$

where $f(x)$ is a given function.

We begin by getting a particular solution of (1) which satisfies condition (2). Assume $V=e^{-\alpha y+\beta x}$ where $\alpha$ and $\beta$ are constants. Hence

$$
\begin{aligned}
& d^{2} v / d x^{2}=\beta^{2} e^{-\alpha y+\beta x} \\
& d^{2} v / d y^{2}=\alpha^{2} e^{-\alpha y+\beta x} .
\end{aligned}
$$


Substituting these values for the derivatives in (1), we obtain

$$
\beta^{2} e^{-\alpha y+\beta x}+\alpha^{2} e^{-\alpha y+\beta x}=0,
$$

or

$$
\beta^{2}+\alpha^{2}=0
$$

If this condition is satisfied, then $v=e^{-\alpha y+\beta x}$ is a solution. Hence

$$
\beta= \pm 1 \alpha \text {. }
$$

So $v=e^{-\alpha y+\beta x}$ is a solution of (1). This form involves an imaginary quantity but we can remedy this. Take $v_{1}=e^{-\alpha y_{e} i \alpha x}$ and $v_{2}=e^{-\alpha y_{e}} e^{-i \alpha x}$ as solutions of (1). Then,

$$
v_{1}=e^{-\alpha y}(\cos \alpha x+i \sin \alpha x)
$$

and

$$
v_{2}=e^{-\alpha y}(\cos \alpha x-i \sin \alpha x) \text {. }
$$

Adding these values of $\mathrm{V}$ and dividing by two, we obtain

$$
v=e^{-\alpha y} \cos \alpha x \text {. }
$$

Next, subtract $v_{2}$ from $v_{1}$ and divide by $2 i$, and we have

$$
v=e^{-\alpha y} \sin \alpha x
$$

We can show that $v=e^{-\alpha y_{c o s} \alpha x}$ and $v=e^{-\alpha y} \sin \alpha x$ are solutions

of (1) and that they also satisfy condition (2).

We need to build up a value of $\mathrm{V}$ which will reduce to 
(4)

$$
f(x)=1 / \pi \int_{0}^{\infty} d \alpha \int_{-\infty}^{\infty} f(\lambda) \cos (\lambda-x) d \lambda,
$$

when $y=0$. Take $e^{-\alpha y} \cos \alpha x$ and $e^{-\alpha} y_{\sin } \alpha x$ and multiply the first by $\cos \alpha \lambda$ and the second by $\sin \alpha \lambda$. Adding these, we obtain

$$
e^{-\alpha y} \cos \alpha(\lambda-x)
$$

This value still satisfies (1). Next, multiply this value by $f(\lambda) \Delta \lambda$ and we have

$$
e^{-\alpha y_{f}(\lambda) \cos \alpha(\lambda-x) \Delta \lambda}
$$

as a value of $\mathrm{V}$ which satisfies (1).

$$
v=\int_{-\infty}^{\infty} e^{-\alpha y_{f}(\lambda) \cos \alpha(\lambda-x) d \lambda}
$$

is still a solution of (1) since it is the limit of the sum of terms of the form (5). Finally,

$$
v=1 / \pi \int_{0}^{\infty} d \alpha \int_{-\infty}^{\infty} e^{-\alpha y_{f}(\lambda) \cos \alpha(\lambda-x) d \lambda}
$$

is a solution of (1) as it is $1 / \pi$ multiplied by the limit of the sum of terms formed by multiplying the right side of (6) by $\mathrm{d} \alpha$ and giving different values to $\alpha$.

The equation (7) will be our solution since it satisfies (1) and (2), and it reduces to (4) when $y=0$ and therefore satisfies condition (3). 


\section{BIBLIOGRA PHY}

1. Byerly, William E.

Fourier Series, Boston:

Ginn \& Company, 1895.

2. Carslaw, H. S.

Theory of Fourier's Series and Integrals, London:

Macmillan and Company, 1921.

3. Churchill, Ruel V.

Fourier Series and Boundary Value Problems, New York:

McGraw-Hil1 Book Company, 1941.

4. Hardy, G. H., and W. W. Rogosinski.

Fourier Series, New York:

The Macmillan Company, 1944.

5. Jackson, Dunham.

Fourier Series and Orthogonal Polynomials, Menasha, Wisconsin: George Bantan Publishing Company, 1941.

6. Symon, Keith R.

Mechanics, London:

Addison-Wesley Publishing Company, 1960.

7. Titchmarsh, E. C.

Theory of Fourier Integrals, 0xford:

Oxford University Press, 1937.

8. Tolstov, Georgi P.

Fourier Series, Englewood Cliffs, New Jersey:

Prentice-Hall, Inc., 1962. 


\section{ACKNOWLEDGMENT}

The author wishes to express his sincere thanks and appreciation to Professor S. Thomas Parker for his helpful suggestions and assistance with the preparation of this report. 
FOURIER SERIES AND THE FOURIER INTEGRAL

by

BILLY GENE CLAYBROOK

B. S., Missouri State Teachers College, 1964

AN ABSTRACT OF A MASTER'S REPORT

submitted in partial fulfillment of the

requirements for the degree

MASTER OF SCIENCE

Department of Mathematics

KANSAS STATE UNIVERSITY

Manhattan, Kansas

1966 
The primary purpose of this Report is to consider some of the most important concepts of Fourier series and Fourier integrals. Most of the periodic functions discussed in this Report are defined and integrable over the interval $[-\pi, \pi]$ and the analysis of the Fourier series is carried out for functions on this interval; however, it is shown that this can be extended to functions defined and integrable over more general intervals such as, $[-\mathrm{L}, \mathrm{L}]$. The analysis consists not only of considering conditions under which a periodic function can be represented by its Fourier series, but also of considering conditions under which a Fourier series may be differentiated and integrated term by term. Conditions are also given for which a nonperiodic function can be represented by its Fourier integral.

When the Fourier series of a function is being studied, the integrands giving rise to the Fourier coefficients of the function are assumed to be integrable in the sense of Riemann. The extension of Riemann integration to the case of improper integrals is made when necessary. This is the case for the Fourier integral of a function. Fourier series and Fourier integrals are interesting in themselves and as a valuable tool in solving physical problems. In this Report various problems are discussed, and the application of Fourier analysis to them is shown. 